\title{
Thermoregulatory Efficiency is Increased after Heat Acclimation in Tropical Natives
}

\author{
Flávio C. Magalhães ${ }^{1), 2)}$, Renata L. F. Passos ${ }^{1)}$, Michele A. Fonseca ${ }^{1)}$, Kenya P. M. Oliveira ${ }^{1)}$, \\ João B. Ferreira-Júnior ${ }^{1)}$, Angelo R. P. Martini ${ }^{1)}$, Milene R. M. Lima ${ }^{1)}$, Juliana B. Guimarães ${ }^{1)}$, \\ Valério G. Baraúna ${ }^{2)}$, Emerson Silami-Garcia ${ }^{1)}$ and Luiz O. C. Rodrigues ${ }^{1)}$ \\ 1) Laboratory of Exercise Physiology, School of Physical Education, Physical Therapy and \\ Occupational Therapy, Department of Physical Education, Federal University of Minas Gerais, \\ Minas Gerais, Brazil \\ 2) Laboratory of Biochemistry of Physical Activity, School of Physical Education and Sport, \\ Department of Biodynamic of the Human Movement, University of São Paulo, Brazil
}

\begin{abstract}
To evaluate the effects of heat acclimation on sweat rate redistribution and thermodynamic parameters, 9 tropical native volunteers were submitted to 11 days of exercise-heat exposures $\left(40 \pm 0^{\circ} \mathrm{C}\right.$ and $45.1 \pm 0.2 \%$ relative humidity). Sudomotor function was evaluated by measuring total and local (forehead, chest, arm, forearm, and thigh) sweat rates, local sweat sodium concentration, and mean skin and rectal temperatures. We also calculated heat production $(\mathrm{H})$, heat storage $(\mathrm{S})$, heat exchange by radiation $(\mathrm{R})$ and by convection $(\mathrm{C})$, evaporated sweat $\left(\mathrm{E}_{\mathrm{sw}}\right)$, sweating efficiency $\left(\eta_{\mathrm{sw}}\right)$, skin wettedness $\left(\mathrm{w}_{\mathrm{sk}}\right)$, and the ratio between the heat storage and the sum of heat production and heat gains by radiation and convection $(\mathrm{S} /(\mathrm{H}+\mathrm{R}+\mathrm{C}))$. The heat acclimation increased the whole-body sweat rate and reduced the mean skin temperature. There were changes in the local sweat rate patterns: on the arm, forearm, and thigh it increased significantly from day 1 to day 11 (all $p<0.05$ ) and the sweat rates from the forehead and the chest showed a small nonsignificant increase ( $p=0.34$ and 0.17 , respectively). The relative increase of local sweat rates on day 11 was not different among the sites; however, when comparing the limbs (arm, forearm, and thigh) with the trunk (forehead and chest), there was a significant higher increase in the limbs $(32 \pm 5 \%)$ in comparison to the trunk $(11 \pm 2 \%$, $p=0.001)$. After the heat acclimation period we observed higher $\mathrm{w}_{\mathrm{sk}}$ and $\mathrm{E}_{\mathrm{sw}}$ and reduced $\mathrm{S} /(\mathrm{H}+\mathrm{R}+\mathrm{C})$, meaning greater thermoregulatory efficiency. The increase in the limb sweat rate, but not the increase in the trunk sweat rate, correlated with the increased $\mathrm{w}_{\mathrm{sk}}, \mathrm{E}_{\mathrm{sw}}$, and reduced $\mathrm{S} /(\mathrm{H}+\mathrm{R}+\mathrm{C})(p<0.05$ to all). Altogether, it can be concluded that heat acclimation increased the limbs' sweat rates in tropical natives and that this increase led to increased loss of heat through evaporation of sweat and this higher sweat evaporation was related to higher thermoregulatory efficiency. J Physiol Anthropol 29(1): 1-12,
\end{abstract}

2010 http://www.jstage.jst.go.jp/browse/jpa2

[DOI: 10.2114/jpa2.29.1]

Keywords: sweat function, heat acclimation, facilitated sweat evaporation

\section{Introduction}

Heat acclimatization involves natural exposure to a hot environment and leads to improved ability to exercise in the heat. Heat acclimation (HA) produces similar responses, but is accomplished in artificially controlled environmental chamber. Heat acclimatization and HA result in similar physiological adaptations if all factors are identical in both environments (Armstrong and Maresh, 1991).

HA is caused by chronic increase in body temperature and leads to numerous adaptations in humans such as decreased heart rate, internal and mean skin temperatures and enhanced sweat secretion that culminates in increased tolerance to exercise in hot environments (Nadel et al., 1974; Patterson et al., 2004; Machado-Moreira et al., 2005; Magalhães et al., 2006). In addition to the increased whole-body sweat production, it has been noted that acclimation to exercise in the heat can change the local sweat rate pattern among body regions, that is, causes sweat redistribution (Patterson et al., 2004; Höfler, 1968; Shvartz et al., 1979).

Fox et al. (1964) were probably the first to notice that during $\mathrm{HA}$, although whole-body sweat rate increased $50 \%$, local arm sweat rate increased $300 \%$ possibly indicating sweat redistribution. However, a more comprehensive evaluation was provided by Höfler (1968) and Shvartz et al. (1979) that observed a higher sweat rate in the limbs in comparison to the 
trunk in response to HA. The authors speculated that this response was because the limbs have a favorable area-to-mass ratio for heat dissipation and a higher sweat rate in the limbs would favor sweat evaporation and therefore thermal homeostasis. More recently, Regan et al. (1996) reported a higher increase in the forearm sweat rate in comparison to the forehead after the HA, strengthening the preferential limb sweat redistribution hypothesis.

However this pattern does not appear to be a universal observation following HA. Cotter et al. (1997) did not observe sweat redistribution after a 6-day HA period and neither did Inoue et al. (1999) after 8 days of HA. The duration of the protocols used in those studies might have been too short to induce local sudomotor adaptation. The hypothesis of the preferential sweat redistribution to the limbs was recently confronted with a well-controlled study (Patterson et al., 2004) in which the authors used a humid and hot environment to acclimate the subjects but did not observe a higher increase in the sweat rate in the limbs. They described that probably the increase in the local sweat rates are due to adaptations of the local sweat gland. For instance, glands below their capacity would show the highest increase in the sweat rate. Therefore, it is still not completely established which effect HA has on local sweat rate adaptation.

Many authors have described sweating differences between tropical and nontropical natives (Thomson, 1954; Kuno, 1956; Fox et al., 1974; Hori et al., 1976; Nguyen and Tokura, 2003). It has been suggested that tropical natives have a more efficient thermoregulatory system (Hori, 1995) relying more on dry heat exchange (radiation and convection) because of higher skin temperature and, therefore, requiring less sweat production and evaporation for temperature control. Nguyen and Tokura (2003) showed that Vietnamese had a higher oscillation in internal temperature in comparison to Japanese, what could lead to less sweat production. Furthermore, Saat et al. (2005a) observed that tropical natives from Malaysia had lower sweat rate in the back, and higher skin and rectal temperatures during rest, exercise and recovery in comparison with Japanese. In addition, Saat et al. (1999) showed that sweat onset time was decreased after Malaysians moved to Japan, and this response was dependent on the time of residence in the temperate region, showing that adaptations acquired due to long stay in a hot region were lost in response to immigration to a cooler region. This evidence indicates that probably tropical natives have a higher set point for internal and skin temperatures than nontropical natives due to residence in a hot environment that enhances dry heat loss.

It has also been pointed out that long-term exposure to passive heat might alter HA responses (Hellon et al., 1956; Wyndham et al., 1952). In fact, a recent study in our laboratory showed that sweating adaptation in tropical natives occurs in a nonclassical manner to HA (Magalhães et al., 2006). Shapiro et al. (1981) observed no difference in sweat rate and rectal temperature in response to HA in the summer, when presumably the subjects were already naturally heat acclimated. Although there are many studies on HA responses in temperate climate inhabitants, HA responses in tropical natives are scarce. Because chronic exposure to heat might induce some adaptations that increase heat endurance in this population, it is interesting to consider how they would respond to a period of HA at the local sweat level. To the best of our knowledge, no studies are available that evaluate this response in tropical natives subjected to a period of HA.

Furthermore, heat-acclimated individuals experience less strain during exercise in the heat than nonacclimated ones (Patterson et al., 2004; Berglund and Gonzalez, 1977). Considering that the thermal load during exercise that an individual has to deal with is the sum of the heat produced and the heat gained by radiation and convection, and the heat stored in the body is the result of the total thermal load minus the heat lost to the environment, then the ratio between heat storage and the sum of heat produced and heat gained by radiation and convection gives a measure of the thermoregulatory efficiency of the individual. We are unaware of any study that has evaluated by which means the human organism deals with a higher thermal load during exercise experienced after adaptation to exercise in the heat.

Thermodynamic computation requires simple data for its applicability and is a helpful tool in evaluating heat exchange in humans since its real measurement requires a calorimetric chamber and is technically difficult to apply. Although simple, we could not find many studies that used these equations to evaluate the main heat exchange avenues in heat-acclimated individuals during exercise.

Therefore, in the present study we evaluated sweat rate redistribution, local sweat sodium concentration, and local skin temperature following HA in tropical natives. In addition, we sought to evaluate, using thermodynamic computations, the main heat-exchange avenues after a period of adaptation to exercise in a hot dry environment. Specifically, we sought to evaluate how the heat-adapted human organism handles a high total thermal load with smaller strain.

\section{Materials and Methods}

\section{Subjects}

After responding to a medical questionnaire and being free of any diseases that could interfere with the results of the study, nine male subjects (age: $25.0 \pm 0.7$ years; weight: $80.48 \pm 1.92 \mathrm{~kg}$; height: $179.6 \pm 1.7 \mathrm{~cm}$; body surface area: $2.00 \pm 0.03 \mathrm{~m}^{2}$ ), inhabitants (from birth to at least 5 years of age and at least 3 years before the study) of a tropical region (Belo Horizonte, Minas Gerais, Brazil, latitude $19.5^{\circ} \mathrm{S}$ and longitude $43^{\circ} \mathrm{W}$ ) volunteered to participate in this study. The study was conducted from June to September (end of fall and winter for the Southern Hemisphere). During the entire period of the experiment, subjects were asked to maintain their habitual diet in order to diminish the effect of food intake variation on the variables measured. The diet content was analyzed using DietPro 3.0. All procedures were in accordance 
with the Helsinki Declaration of 1975, and the Ethics Committee of research of the Federal University of Minas Gerais, Brazil, approved all procedures; written and informed consent was obtained from all the subjects.

\section{Heat acclimation protocol}

HA was accomplished by a total of 11 days of 1 hour/day exercise in the heat using a controlled-hyperthermia technique in which the work rate is modified in order for the subjects to maintain a target internal temperature during a given period of time (Patterson et al., 2004; Cotter et al., 1997; Fox et al., 1961, 1967). HA was accomplished by elevating rectal temperature by $1^{\circ} \mathrm{C}$ in a 30 -min treadmill exercise $(1 \%$ grade and $2.20 \pm 0.11 \mathrm{~m} \cdot \mathrm{s}^{-1}$ ) and maintaining it elevated by another $30 \mathrm{~min}\left(1.69 \pm 0.11 \mathrm{~m} \cdot \mathrm{s}^{-1}\right)$ (Regan et al., 1996) in $40 \pm 0^{\circ} \mathrm{C}$ and $45.1 \pm 0.2 \%$ relative humidity (rh). Wind speed was set at $1.4 \pm 0.1 \mathrm{~m} \cdot \mathrm{s}^{-1}$ and was provided by an electric fan (Arno SL40, series MH, 120W, São Paulo, Brazil). This environment was chosen based on previous studies that were successful in inducing HA (Shvartz et al., 1979; Regan et al., 1996). The duration of the acclimation period was $13 \pm 0.4$ days (range 12 15 days).

\section{Body temperature}

Each day the subjects were instructed to void their bladders and insert a rectal temperature probe (Yellow Spring Instruments, Series 400). Skin temperature probes (Yellow Spring Instruments, Series 400A) were attached to three skin sites: chest, arm, and thigh and mean skin temperature was calculated as following (Roberts et al., 1977): $0.43 \cdot \mathrm{T}_{\text {chest }}$ $+0.25 \cdot \mathrm{T}_{\text {arm }}+0.32 \cdot \mathrm{T}_{\text {thigh }}$. Mean body temperature was calculated as $0.8 \cdot$ rectal temperature $+0.2 \cdot$ mean skin temperature (Stolwijk and Hardy, 1966). Heart rate (HR) was measured continuously (Polar Vantage NV, Kempele, Finland). Subjects were then moved to a climatic chamber (Russels Technical Products, WMD 1150-5, Holland, MI, USA) and rested for $5 \mathrm{~min}$ in a seated position while the baseline measurements were taken.

\section{Local and total sweat rate and sweat sodium content}

On days 1, 6, and 11 of HA we measured local sweat rates at 5 skin sites: forehead (middle of the forehead), chest (right side, between the nipple line and the clavicle), arm (upper right arm), forearm (proximal third of the anterior right forearm) and thigh (right mid-thigh) using the absorbent filter paper patches technique (Vimieiro-Gomes et al., 2005; Saat et al., 2005b) during the entire second half of the exercise protocol (for $30 \mathrm{~min}$ ). On adjacent skin regions to where we measured local sweat rate, we also measured local skin temperature. Sweat sodium concentration was measured according to Kirby and Convertino (1986) with slight modifications: the sweat collected by the absorbent filter paper was calculated and diluted 50 times with distilled water. Sweat sodium concentration was measured by flame spectrophotometry.
Whole-body sweat rate $\left(\dot{\mathrm{M}}_{\mathrm{sw}}\right)$ was calculated by the difference in pre- and post-exercise nude body weights (Filizola ${ }^{\circledR}$ MF-100 scale, precision of $0.02 \mathrm{~kg}$, São Paulo, SP, Brazil), normalized by body surface area $\left(\mathrm{A}_{\mathrm{D}}\right.$, (Dubois and Dubois, 1916)) and divided by time, uncorrected for metabolic or respiratory variations.

\section{Aerobic capacity assessment}

Four to five days before the start and after the end of the HA period, the subjects performed an aerobic capacity test in a thermoneutral environment $\left(23 \pm 0^{\circ} \mathrm{C}\right.$ and $59.2 \pm 0.4 \%$ relative humidity). This temperature was chosen because aerobic capacity is diminished in the heat and we did not want to have this confounding effect on this variable. These tests involved walking/running on a treadmill starting with 3 MET intensity followed by increases in the speed and grade that resulted in increases in 3 MET every 3 minutes until volitional exhaustion (Fielding et al., 1997). Exhaustion was defined when subjects could no longer maintain the predetermined speed, or voluntarily stopped running. If heart rate was not higher than the maximal heart rate predicted by age and if $\dot{\mathrm{V}}_{\mathrm{CO}_{2}} / \dot{\mathrm{V}}_{\mathrm{O}_{2}}$ was lower than 1.1, the test was not considered and the subject was excluded. Expired gases were collected in a gas analyzer chamber and analyzed breath-by-breath to derive peak oxygen consumption $\left(\dot{\mathrm{VO}}_{2 \text { peak }}\right)$ by means of a gas analyzer (BIOPAC System MP35) previously calibrated using a standard gas mixture. The $\dot{\mathrm{VO}}_{2}$ from the last minute of exercise was assumed as $\dot{\mathrm{VO}}_{2 \text { peak }}$.

\section{Hydration status}

To guarantee the euhydrated state and normal sweating responses, subjects were instructed to drink $500 \mathrm{~mL}$ of pure tap water 2 hours before each experimental day began. Hydration status was assessed by urine specific gravity $\left(\mathrm{U}_{\mathrm{g}}\right)$ both before and after each experimental day session using a portable refractometer (JSCP-Uridens, São Paulo, SP, Brazil) previously calibrated with distilled water. The subjects were considered euhydrated when $\mathrm{U}_{\mathrm{g}}<1.030$ (Armstrong, 2000). During the acclimation period water intake was provided $a d$ libitum. In all experiments subjects wore shorts, socks, and running shoes.

\section{Thermodynamic equations}

In order to assess the heat exchange parameters in days 1, 6, and 11 of the acclimation period, we used the set of thermodynamic equations. Heat production $(\mathrm{H})$ was calculated as (Nielsen, 1996): $\mathrm{H}=(4 \cdot v \cdot \mathrm{m}) \cdot \mathrm{A}_{\mathrm{D}}^{-1}$, where, $v$ is the running speed in $\mathrm{m} \cdot \mathrm{s}^{-1}, m$ is the subject mass in $\mathrm{kg}$ and $\mathrm{A}_{\mathrm{D}}$ is the body surface area in $\mathrm{m}^{2}$. Heat storage (S) can be calculated as (Nielsen, 1996): $\mathrm{S}=(3480) \cdot\left(\mathrm{m} \cdot \Delta \overline{\mathrm{T}}_{\mathrm{b}} / \mathrm{t}\right) \cdot \mathrm{A}_{\mathrm{D}}^{-1}$, where 3480 is the specific heat of body tissues (in $\mathrm{J} \cdot \mathrm{kg}^{-1} \cdot{ }^{\circ} \mathrm{C}^{-1}$ ) and $\Delta \overline{\mathrm{T}}_{\mathrm{b}}$ is the change in mean body temperature over the exercise period $(t)$ in seconds. The heat exchange by radiation $(\mathrm{R})$ and convection (C), in $\mathrm{W} \cdot \mathrm{m}^{-2}$, were calculated according to Nielsen (1996): $\mathrm{R}=\left(\left(\overline{\mathrm{T}}_{\mathrm{sk}}-\mathrm{T}_{\mathrm{ra}}\right) \cdot 5.2\right) \cdot \mathrm{A}_{\mathrm{D}}^{-1} \quad$ and $\quad \mathrm{C}=\left(\left(\mathrm{T}_{\mathrm{sk}}-\mathrm{T}_{\mathrm{a}}\right) \cdot \mathrm{v}^{0.5} \cdot 8.3\right) \cdot \mathrm{A}_{\mathrm{D}}^{-1}$; 
where $T_{r a}$ is the mean radiant temperature of the walls of the climatic chamber, $\mathrm{T}_{\mathrm{a}}$ is the ambient temperature, and 5.2 and 8.3 are the heat coefficients (in $\mathrm{W} \cdot \mathrm{m}^{-2} \cdot{ }^{\circ} \mathrm{C}^{-1}$ ) for radiant and convective heat exchange, respectively. Evaporated sweat $\left(\mathrm{E}_{\mathrm{sw}}\right)$ can be calculated as the residual component from: $\mathrm{E}_{\mathrm{sw}}=\mathrm{H} \pm$ $\mathrm{R} \pm \mathrm{C} \pm \mathrm{S}$. Sweating efficiency $\left(\eta_{\mathrm{sw}}\right)$ was calculated by the ratio of $E_{s w}$ to $\dot{M}_{s w}$, or the percentage of sweat evaporated from the sweat produced (Frye and Kamon, 1983). Skin wettedness $\left(\mathrm{w}_{\mathrm{sk}}\right)$ was calculated as the ratio between $\mathrm{E}_{\mathrm{sw}}$ and the evaporative capacity of the environment (Candas et al., 1979a). Thermoregulatory efficiency was assumed to be the ratio of the accumulated heat(S)-to-the total heat load $(\mathrm{H}+\mathrm{R}+\mathrm{C})$.

All thermodynamic parameters calculated were from the entire period of exercise, i.e., $60 \mathrm{~min}$.

\section{Statistical analyses}

Two-way repeated measures ANOVA was used to compare local sweat rate values, body temperatures, and heart rate, and one-way repeated measures ANOVA was used to compare calculated thermodynamic values. When a significant $F$ value was found we performed a Student Newman Keuls as a posthoc. Regression analyses curves were constructed and the $r$ value calculated. Pearson's correlation was used to evaluate the significance. The $\alpha$ level was set at .05. Data are shown as mean \pm standard error of the mean (SEM) unless otherwise stated.

\section{Results}

Table 1 shows that maximal heart rate, peak oxygen consumption, maximal rating of perceived exertion, and maximal ratio $\dot{\mathrm{V}}_{\mathrm{CO}_{2}} / \dot{\mathrm{V}}_{\mathrm{O}_{2}}$ were not different between the pre- and post-acclimation aerobic tests and that subjects reached maximal effort during them. Body weight after acclimation $(79.74 \pm 1.91 \mathrm{~kg})$ was not different in comparison to before acclimation $(80.48 \pm 1.92 \mathrm{~kg} ; p=0.86)$.

HA was confirmed following the 11 days of exposure by reduced resting heart rate, increased whole-body sweat rate, and decreased average exercise mean skin temperature $(p<0.05$ to all; Table 2$)$.
Local sweat rate in days 1, 6, and 11 of the acclimation period is shown in Fig. 1A. None of the analyzed sites showed significant increments in local sweat rate by day $6(p>0.05)$. However, local sweat rate from the arm, the forearm, and the thigh were increased by day 11 in comparison to day 1 $(p<0.05)$, while local sweat rate from the forehead and the chest were not altered ( $p=0.34$ and 0.17 , respectively).

Regional sweat rate redistribution is shown in Fig. 1B. There was no difference in the increase in local sweat rates among the sites analyzed. Stratified to the limbs versus the trunk, however, we found a higher increase in the limbs (limbs: $132 \pm 5 \%$ and trunk: $112 \pm 2 \% ; p=0.001)$. Figure $1 \mathrm{C}$ shows that local sweat sodium concentration was not different between days in all sites. However, as sweat rate influences sweat sodium concentration, we divided the local sweat sodium concentration by the local sweat rate, in order to evaluate sweat sodium concentration to a given sweat rate. As shown in Fig. $1 \mathrm{D}$ we found that the local sweat sodium concentration per local sweat rate was reduced in the arm on day 6 in comparison to day $1(p<0.05)$, and a further decrease on day 11 in comparison to day $6(p<0.05)$. In the forearm and the thigh there was only a significant reduction on day 11 in comparison to day $1(p<0.05)$.

Figure 2 shows the changes in local sweat rate and in the percentage contribution of the different surface areas during the heat acclimation protocol. It can be observed that there was an increase in arm, forearm, and thigh contribution to local sweat production. Furthermore, the relative contribution of the limbs increased from $49.8 \%$ to $52.9 \%(p<0.05)$, while the

Table 1 Maximal exercise heart rates, peak oxygen consumption, maximal exercise rating of perceived exertion and $\dot{\mathrm{V}}_{\mathrm{CO}_{2}} / \dot{\mathrm{V}}_{\mathrm{O}_{2}}$ during aerobic capacity tests before (pre) and after (post) HA

\begin{tabular}{lcc}
\hline & Pre & Post \\
\hline Maximal heart rate $\left(\mathrm{b} \cdot \mathrm{min}^{-1}\right)$ & $190 \pm 1$ & $187 \pm 2$ \\
Resting oxygen consumption $\left(\mathrm{mL} \cdot \mathrm{kg} \cdot{ }^{-1} \cdot \mathrm{min}^{-1}\right)$ & $4.9 \pm 0.4$ & $4.8 \pm 0.3$ \\
Peak oxygen consumption $\left(\mathrm{mL} \cdot \mathrm{kg} \cdot{ }^{-1} \cdot \mathrm{min}^{-1}\right)$ & $50.5 \pm 0.9$ & $52.4 \pm 1.1$ \\
Maximal rating of perceived exertion (units) & $19 \pm 0.6$ & $19 \pm 0.4$ \\
Maximal $\dot{\mathrm{V}}_{\mathrm{CO}_{2}} / \dot{\mathrm{O}}_{2}$ & $1.15 \pm 0.05$ & $1.16 \pm 0.05$
\end{tabular}

Table 2 Resting and mean exercise heart rate, mean exercise rate of perceived exertion, mean power output, whole-body sweat rate, average exercise rectal temperature, average exercise average skin temperature, and mean exercise mean body temperature on days 1, 6, and 11 of $\mathrm{HA}$

\begin{tabular}{lccc}
\hline & Day 1 & Day 6 & Day 11 \\
\hline Resting heart rate $\left(\mathrm{b} \cdot \mathrm{min}^{-1}\right)$ & $80 \pm 3$ & $75 \pm 4$ & $74 \pm 4 *$ \\
Mean exercise heart rate $\left(\mathrm{b} \cdot \mathrm{min}^{-1}\right)$ & $144 \pm 5$ & $137 \pm 6$ & $143 \pm 5$ \\
Mean exercise rating of perceived exertion (units) & $12 \pm 1$ & $71 \pm 1$ & $842 \pm 25^{*}$ \\
Mean power output $(\mathrm{W})$ & $755 \pm 23$ & $785 \pm 28$ & $8.5 \pm 0.4^{*}$ \\
Whole-body sweat rate $\left(\mathrm{g} \cdot \mathrm{m}^{-2} \cdot \mathrm{min}^{-1}\right)$ & $37.7 \pm 0.3$ & $37.27 \pm 0.4$ & $37.32 \pm 0.19$ \\
Resting rectal temperature $\left({ }^{\circ} \mathrm{C}\right)$ & $38.03 \pm 0.13$ & $38.06 \pm 0.12$ & $35.54 \pm 0.12$ \\
Average exercise rectal temperature $\left({ }^{\circ} \mathrm{C}\right)$ & $35.84 \pm 0.25$ & $35.55 \pm 0.27^{*}$ & $37.57 \pm 0.09$ \\
Average exercise mean skin temperature $\left({ }^{\circ} \mathrm{C}\right)$ & $37.56 \pm 0.10$ & $37.56 \pm 0.09$ & \\
Average exercise mean body temperature $\left({ }^{\circ} \mathrm{C}\right)$ & & & \\
\hline
\end{tabular}

$* p<0.05$ in comparison with day 1 

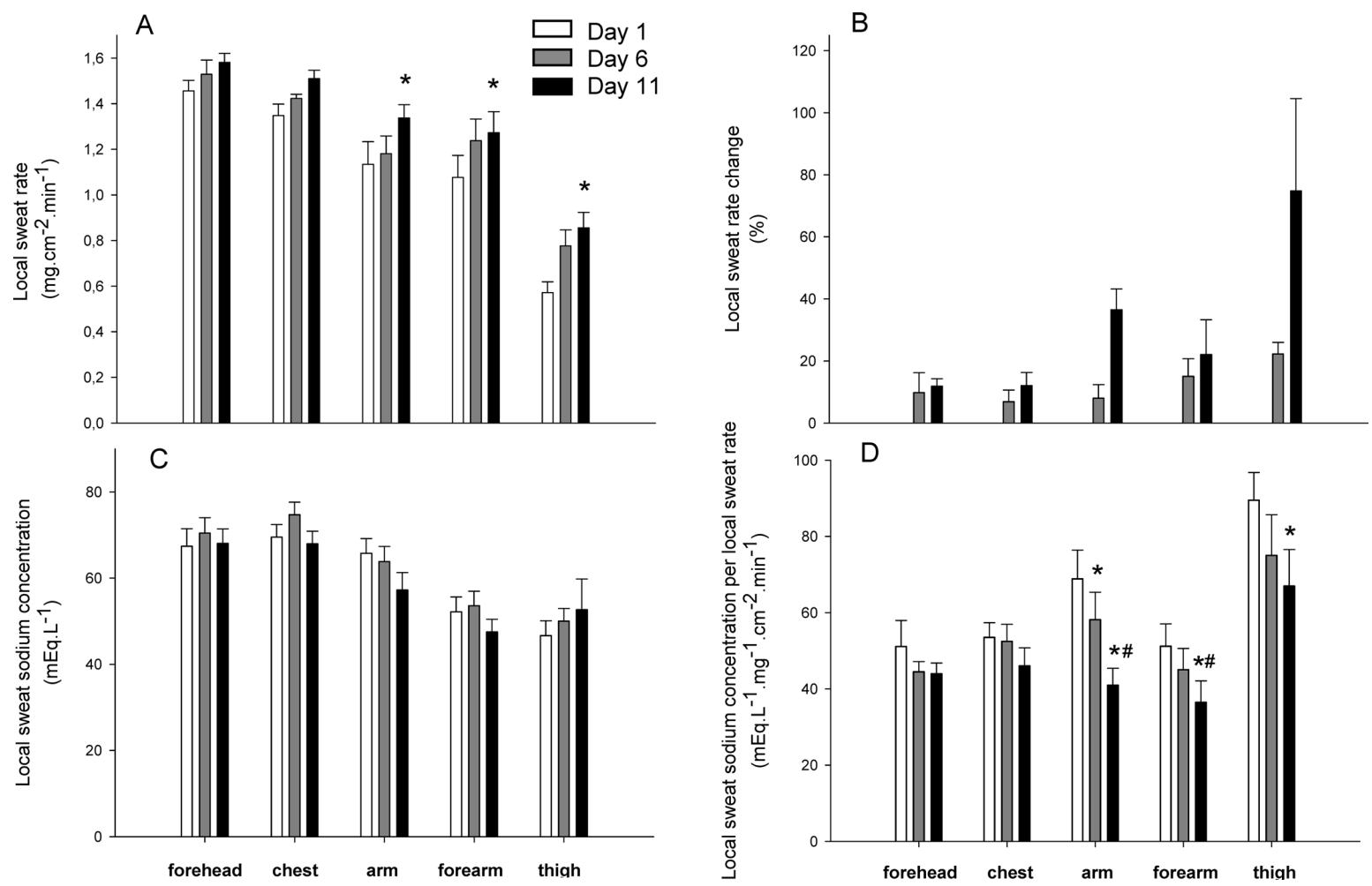

Fig. 1 Local sweat rates (A), relative elevations in local sweat rates (B), local sweat sodium concentration (C), and local sweat sodium concentration per local sweat rate (D) on the forehead, the chest, the arm, the forearm, and the thigh on days 1, 6 and 11 of heat acclimation. $* p<0.05$ in comparison to day 1 and $\# p<0.05$ in comparison to day 6 .

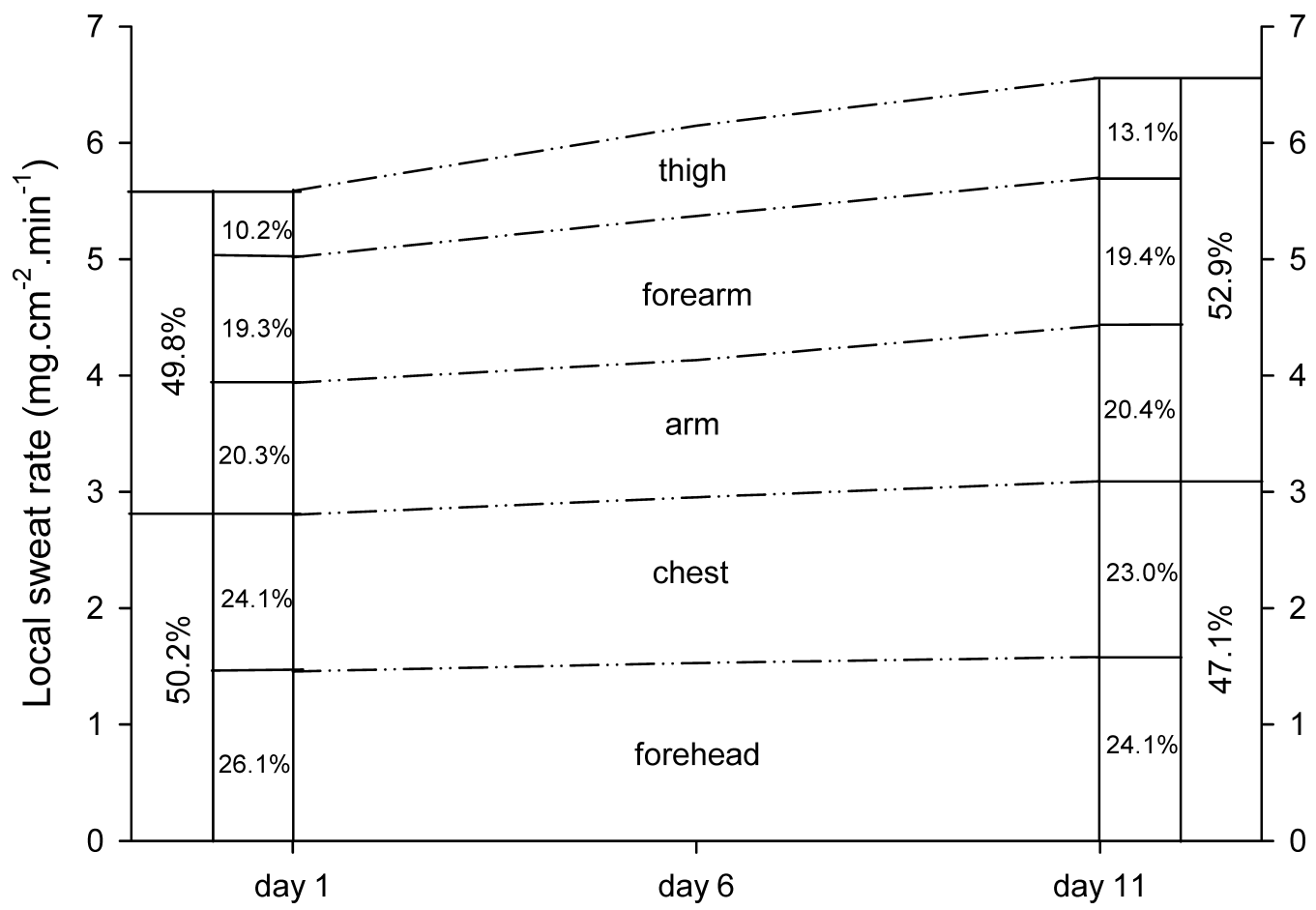

Fig. 2 Changes in local sweat rate and in percentage contribution of the different surface areas during the heat acclimation protocol. 

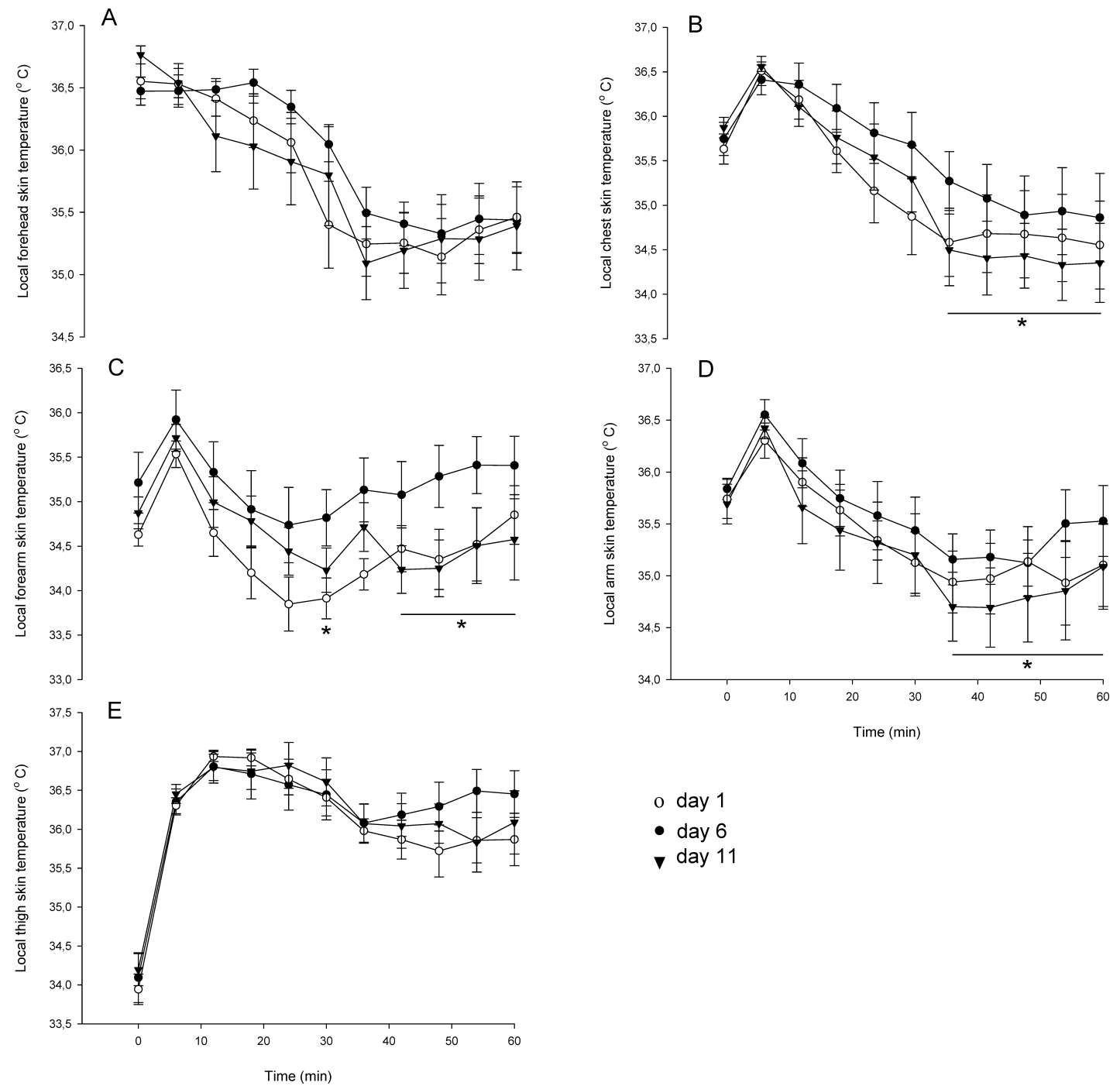

Fig. 3 Local skin temperatures on the forehead (A), the chest (B), the arm (C), the forearm (D), and the thigh (E) on days 1, 6, and 11 of heat acclimation. ${ }^{*} p<0.05$ day 11 in comparison to day 1 .

relative contribution of the forehead and the chest decreased from $50.2 \%$ to $47.1 \%(p<0.05)$.

The core-to-skin temperature gradient was increased on day 11 compared to day 1 (day $1: 2.10 \pm 0.24^{\circ} \mathrm{C}$ and day $11: 2.45 \pm$ $\left.0.30^{\circ} \mathrm{C}, p<0.05\right)$. This gradient is a measure of the thermal conductance of the body shell (Kenney et al., 1990).

Local skin temperature is shown in Fig. 3. There were no differences among days in the forehead temperature $(p<0.05$, Fig. 3A). However, the chest, the arm, and the forearm skin temperatures were lower on day 11 in comparison to day 1 from 36 minutes of exercise to the end of the protocol $(p<0.05$ to all; Figs. 3B, C and D, respectively). The thigh skin temperature was not different among days 1,6 and 11 $(p<0.05$; Fig. 3E).

The diet content was within normal values and did not vary

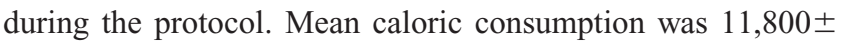
$300 \mathrm{~kJ} /$ day, $55 \pm 0.9 \%$ carbohydrates, $16 \pm 0.5 \%$ proteins, $29 \pm$ $0.9 \%$ fat, $1980 \pm 97 \mathrm{mg}$ of sodium.
Table 3 shows the thermodynamic parameters calculated in days 1,6 , and 11 of HA. Greater $\mathrm{H}$ in day 11 was found due to the higher running speed of the subjects required to maintain the $1^{\circ} \mathrm{C}$ increase in rectal temperature $(p<0.05)$. There was a greater heat gain from $\mathrm{R}$ and $\mathrm{C}$ because of a reduced $\overline{\mathrm{T}}_{\mathrm{sk}}$ after the adaptation period $(p<0.05)$. There was no significant change in $\mathrm{S}$ following the adaptation period (Table 3, $p=0.086$ ). As the $\mathrm{H}, \mathrm{R}$, and $\mathrm{C}$ were increased, the accumulated heat-to-total heat load ratio was reduced (Table 3), meaning a higher thermoregulatory efficiency. $\mathrm{E}_{\mathrm{sw}}$ and $\mathrm{w}_{\mathrm{sk}}$ were higher after the adaptation period ( $p<0.05$ to both). Nonevaporated sweat, calculated as the difference between $\dot{\mathrm{M}}_{\mathrm{sw}}$ and $\mathrm{E}_{\mathrm{sw}}$, was also greater $(0.15 \pm 0.04$ and $0.26 \pm 0.05$ liters on days 1 and 11 , respectively, $p<0.05)$. However, even with a greater nonevaporated sweat, we did not observe a significant difference in $\eta_{\mathrm{sw}}(p=0.073$, Table 3$)$.

The increase in whole-body sweat rate led to increased $\mathrm{w}_{\mathrm{sk}}(r=0.63 ; p<0.01$, Fig. $4 \mathrm{~A})$ after the adaptation to exercise 
Table 3 Heat production, heat exchange by convection and radiation, heat storage, evaporated sweat, sweating efficiency, skin wettedness, and thermoregulatory efficiency on days 1,6 and 11 of the HA

\begin{tabular}{|c|c|c|c|c|c|c|c|c|}
\hline & $\mathrm{H}\left(\mathrm{W} \cdot \mathrm{m}^{-2}\right)$ & $\mathrm{C}\left(\mathrm{W} \cdot \mathrm{m}^{-2}\right)$ & $\mathrm{R}\left(\mathrm{W} \cdot \mathrm{m}^{-2}\right)$ & $\mathrm{S}\left(\mathrm{W} \cdot \mathrm{m}^{-2}\right)$ & $\mathrm{E}_{\mathrm{sw}}\left(\mathrm{W} \cdot \mathrm{m}^{-2}\right)$ & $\eta_{\mathrm{sw}}(\%)$ & $\mathrm{w}_{\mathrm{sk}}(\%)$ & $\mathrm{S} /(\mathrm{H}+\mathrm{C}+\mathrm{R})(\%)$ \\
\hline Day 1 & $301.2 \pm 7.4$ & $-41.9 \pm 1.4$ & $-22.7 \pm 0.9$ & $33.0 \pm 3.2$ & $332.8 \pm 7.1$ & $81.5 \pm 0.0$ & $84.2 \pm 3.2$ & $9.0 \pm 0.9$ \\
\hline Day 6 & $313.2 \pm 9.8$ & $-43.0 \pm 2.0$ & $-23.0 \pm 1.0$ & $30.3 \pm 2.8$ & $348.8 \pm 11.4^{*}$ & $80.5 \pm 0.0$ & $88.3 \pm 3.5$ & $8.1 \pm 0.9$ \\
\hline
\end{tabular}

$\mathrm{H}$, heat production; $\mathrm{C}$, convection; $\mathrm{R}$, radiation; $\mathrm{S}$, heat storage; $\mathrm{E}_{\mathrm{sw}}$, evaporated sweat rate; $\eta_{\mathrm{sw}}$, sweating efficiency; $\mathrm{w}_{\mathrm{sk}}$, skin wettedness; $\mathrm{S} /(\mathrm{H}+\mathrm{C}+\mathrm{R})$, ratio between accumulated heat-to-total heat load. ${ }^{*} p<0.05$ in comparison with day $1 ;{ }^{*} p<0.05$ in comparison to day 6 .

A

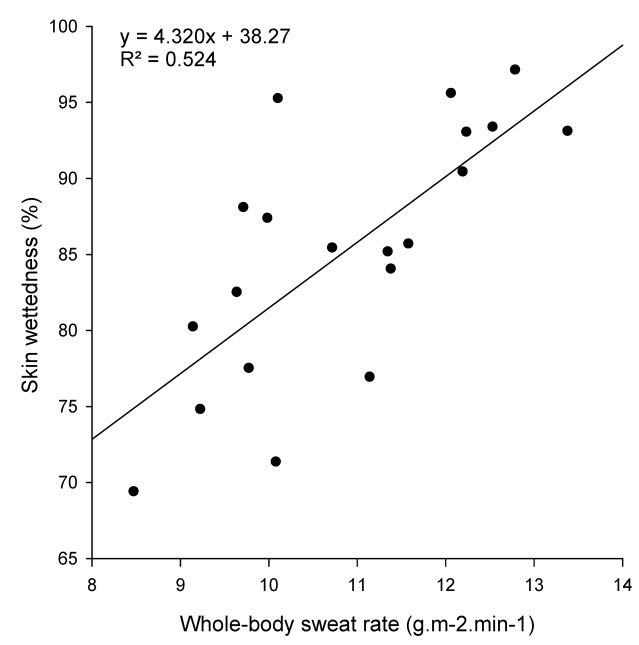

$\mathrm{C}$

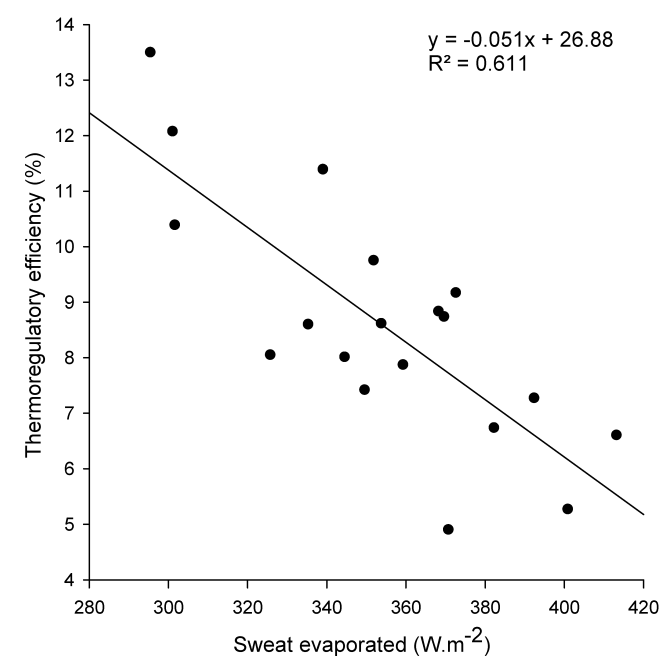

B

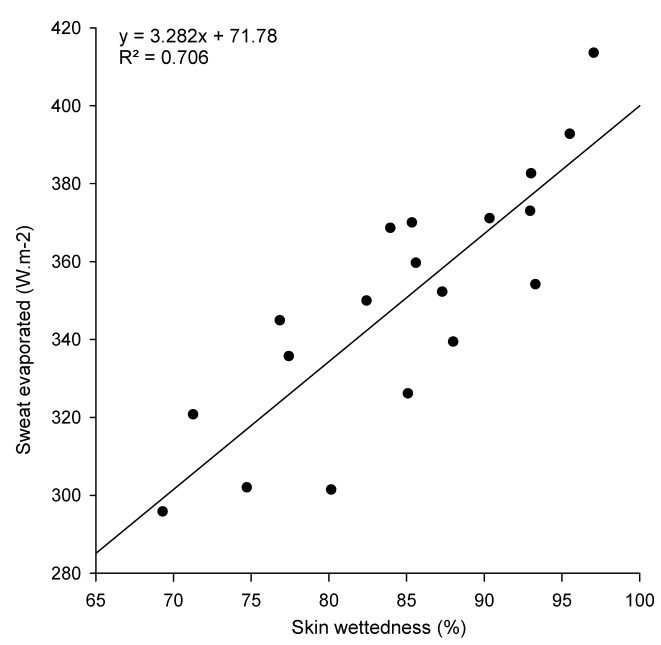

Fig. 4 Correlation between whole-body sweat rate and skin wettedness (A), skin wettedness and sweat evaporation (B), and sweat evaporation and thermoregulatory efficiency $(\mathrm{C})$.

in the heat. The higher $\mathrm{w}_{\mathrm{sk}}$ on day 11 accounted for the higher $\mathrm{E}_{\mathrm{sw}} \quad(r=0.86 ; p<0.0001$, Fig. 4B). The increase in thermoregulatory efficiency was related to increase in $\mathrm{E}_{\mathrm{sw}}$ (Fig. 4C, $r=-0.8 ; p=0.0002$ ).

Interestingly, the limb sweat rate, but not the trunk, was correlated with the increase in $\mathrm{w}_{\text {sk }}$ (Fig. 5A, $r=0.75, p>0.01$ and Fig. 5B, $r=0.08, p>0.05$ ), with the increase in sweat evaporation (Fig. 5C, $r=0.59, p<0.01$ and Fig. 5D, $r=0.2$, $p>0.05$ ), and with the increase in thermoregulatory efficiency (Fig. 5E, $r=-0.73, p<0.01$ and Fig. $5 \mathrm{~F}, r=-0.14, p<0.05$ ). 
A

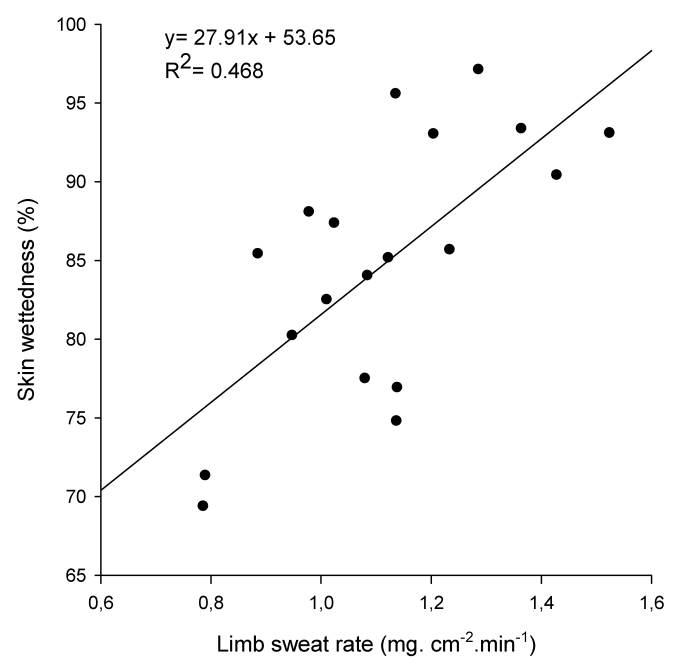

C
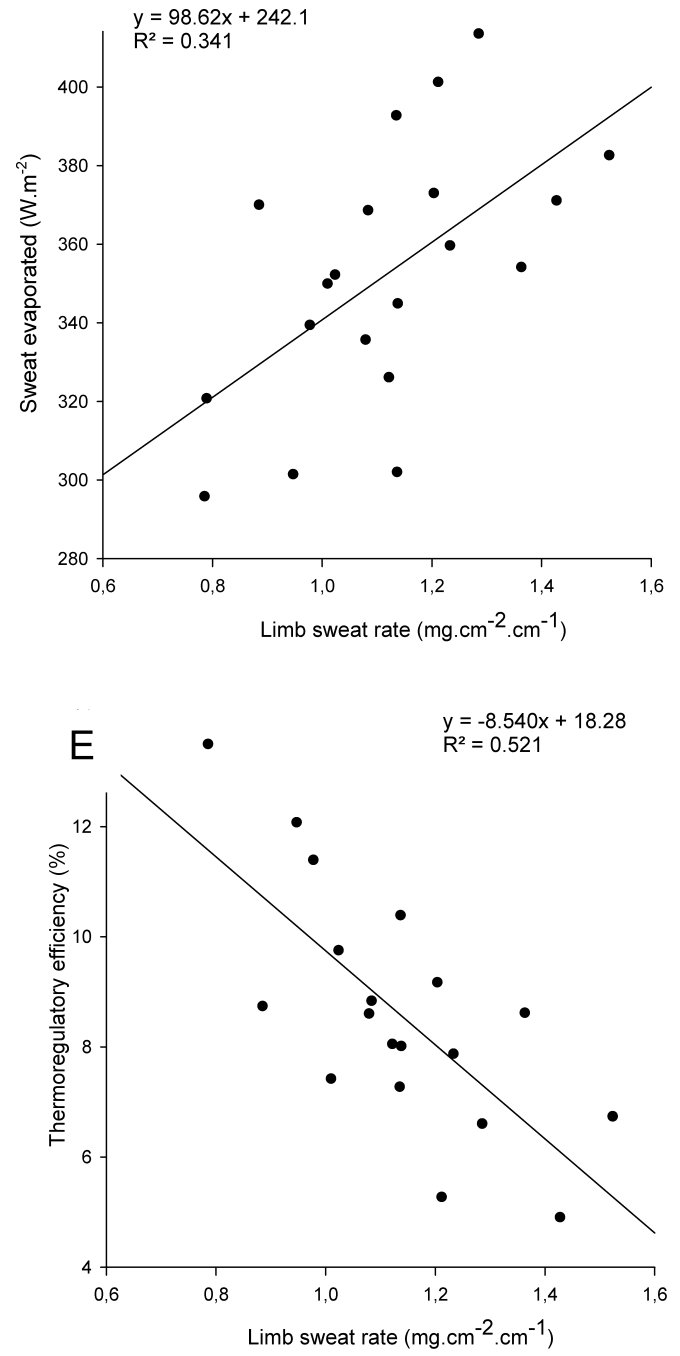

B

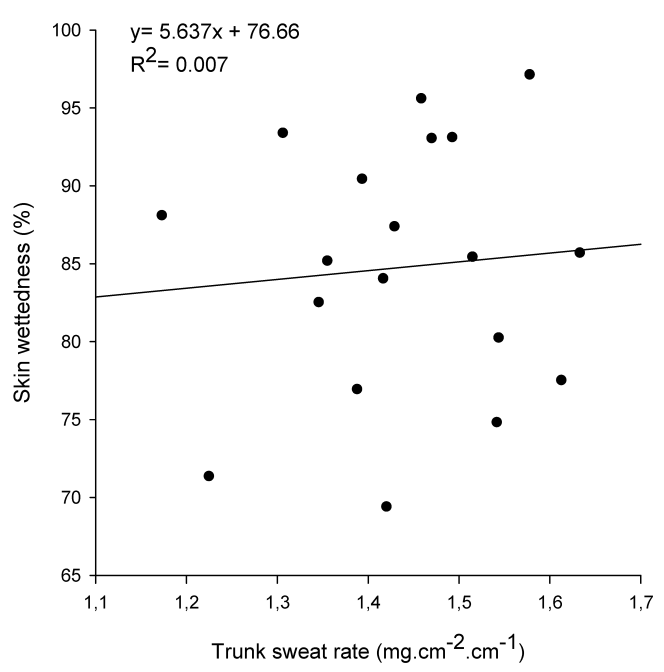

D
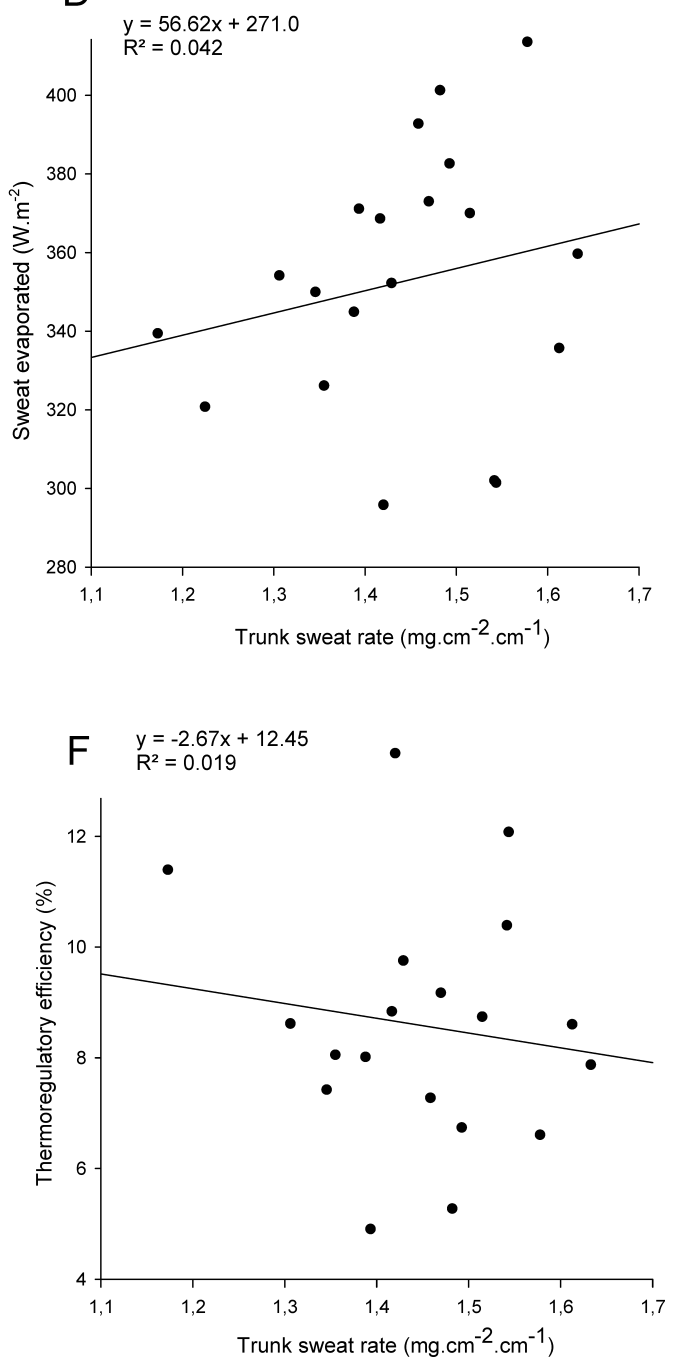

Fig. 5 Correlation between the limb (A) and the trunk (B) sweat rate and skin wettedness; the limb (C) and the trunk (D) sweat rate and evaporated sweat; and the limb (E) and the trunk (F) sweat rate and thermoregulatory efficiency. 


\section{Discussion}

The main and novel findings of the present study are as follows: 1) HA elicits local sweat adaptations in tropical natives, with a preferential increase in local sweat rate in the limbs in comparison to the trunk and a reduction in sweat sodium concentration in the limbs; 2) after the acclimation period there was a higher percentage of the skin covered by sweat (skin wettedness), which may be responsible for the higher sweat evaporation rate with no difference in sweating efficiency; and 3) local skin temperature was reduced during exercise in the chest, the arm, and the forearm on day 11 in comparison to day 1 , showing that probably sweat evaporation on these sites is increased after HA.

In this study the regional sweat pattern changed: in general, the local sweat rate increases, and we found this to be true in the arm, the forearm, and the thigh on day 11 in comparison to day 1 . We also observed a tendency for sweat redistribution towards the limbs: the sweat rate from the thigh, the forearm, and the arm showed a significant increase of $70 \%, 24 \%$, and $35 \%(p<0.05$ to all), respectively; on the other hand, the forehead and the chest did not show significant changes $(11 \%$ and $12 \%$ increases, $p>0.05$ to both, respectively). Shvartz et al. (1979) also used a constant work rate $\left(50 \% \mathrm{~V}_{2 \max }\right)$ in the heat $\left(40{ }^{\circ} \mathrm{C}\right.$ and $\left.48 \% \mathrm{rh}\right)$ for eight days and found sweat redistribution towards the limbs: the increase in the arms and the thigh was higher than that of the chest. Regan et al. (1996) used an environment similar to the present study and observed an increase in the forearm sweat rate in comparison to the forehead, while Patterson et al. (2004) studied the effects of a period of humid HA on sweat redistribution and observed that although there was sweat redistribution, it did not follow the trunk-to-limb pattern. They suggested that those sites where sweat glands are below their capacity show the highest increase in sweat rate. Our results partially support this hypothesis. Regions that showed the smallest sweat rates on day 1 were the ones that presented a significant increase on day 11, while those that were probably closer to their capacity did not show any increase.

In the present study we found an increased sweat rate in the limbs in comparison to the trunk (sweat redistribution) and increased skin wettedness. There has been previously observed a relationship between local sweat rate redistribution and skin wettedness following HA (Candas et al., 1979a) and in the present study there was a positive significant correlation between these two parameters. Furthermore, the increase in the limb sweat rate was correlated with the increase in sweat evaporation and thermoregulatory efficiency. These data suggest that sweat redistribution in response to HA is an important adaptation to increase heat tolerance during exercise in the heat.

There are reports of diminished sweating efficiency after HA due to higher skin wettedness (Kolka et al., 1984; Candas et al., 1979a). In the present study we found an increase in skin wettedness probably because of the sweat redistribution.
However, contrary to previous studies that investigated HA in nontropical natives, we did not observe a difference in sweating efficiency. This difference may be due to the fact that our subjects were partially adapted to heat and when exposed to a period of artificial acclimation increased sweat evaporation and maintained the same sweating efficiency. The precise mechanism responsible for this difference is not clear and has not been addressed in the present study; however, increases in sweat rate can occur by increasing the number of active sweat glands, sweat gland output, or a combination of both. Peter and Wyndham (1966) showed that the maximal number of activated sweat glands does not change in response to a period of HA in nontropical natives; while Kuno (1956) has shown that the number of active sweat glands is higher in tropical natives in comparison to nontropical natives. Therefore, it is possible that one of the adaptations elicited from chronic exposure to heat is the increase in the number of activated sweat glands and that this adaptation could induce higher sweat evaporation for a given sweat output in comparison to increasing sweat gland output. However, as we did not measure the number of activated sweat glands, we can only speculate on this mechanism and it would be interesting to investigate the acclimation to heat in tropical natives at the glandular level in future studies.

We observed higher $\dot{\mathrm{M}}_{\mathrm{sw}}$ after the adaptation period, a finding recurrent in the literature (Patterson et al., 2004; Machado-Moreira, 2005). Increased $\dot{\mathrm{M}}_{\mathrm{sw}}$ is partly responsible for the higher $\mathrm{w}_{\mathrm{sk}}$ and $\mathrm{E}_{\mathrm{sw}}$ also observed, (Mitchell et al., 1976; Candas et al., 1979b); however, as shown by others (Mitchell et al., 1976), $\dot{M}_{\mathrm{sw}}$ was also related to an increase in the nonevaporated sweat. Belding and Hatch (1955) have stated that nonevaporated sweat should not be considered physiologically inefficient, because oversweating is necessary to satisfy the $\mathrm{w}_{\mathrm{sk}}$ requirements, which, in turn, has been found to be responsible for the higher $\mathrm{E}_{\mathrm{sw}}$ (Berglund and Gonzalez, 1977). Candas et al. (1979b) suggested, therefore, that the role of $\mathrm{HA}$ is to develop the required $\mathrm{w}_{\mathrm{sk}}$ for sweat evaporation. However, despite the increase in nonevaporated sweat, we did not find a reduction in $\eta_{\mathrm{sw}}$. Differently, Candas et al. (1979b) described an augmented drippage of around $30 \%$ to $65 \%$ of the sweat produced being wasted while Mitchell et al. (1976) found a $200 \%$ increase in sweat drippage, which led to lower $\eta_{\mathrm{sw}}$. These differences might be related to the higher evaporative capacity of the environment employed in the present study. Thus, we showed that when the environment is suitable for sweat evaporation, $\mathrm{E}_{\mathrm{sw}}$ and $\mathrm{w}_{\mathrm{sk}}$ are enhanced with no concomitant decrease in sweating efficiency.

Lower local skin temperature as observed in the present study after HA means that more sweat was evaporated. It is interesting to note that following HA we observed significantly reduced skin temperature in the chest, the arm, and the forearm and no significant difference in the forehead and the thigh $(p>0.05)$. As the sweat rate increases, one could expect higher sweat evaporation as well and this was the case for the arm and the forearm but not for the thigh. The reason for that is 
unknown but it could be reasoned that the arm and the forearm increased its local sweat rate by means of a higher number of activated sweat glands. Furthermore, the diminished evaporative rate in the thigh could be related to a lower convection rate due to its lower position in the body (Porter, 1993) or because the increase in sweat rate observed was due to an increase in the sweat output per gland. In addition, it is known that convective heat transference is dependent upon the radius of the body segment (Ducharme et al., 1991) so the thigh heat coefficient transfer is lower than the arm and forearm, which might lead to lower sweat evaporation in the thigh compared to the arm and forearm, even though its local sweat rate increased during HA. Interestingly, although the chest did not show an increase in sweat rate, its local skin temperature was reduced after the acclimation period, showing that there was more sweat evaporation but no increase in sweat production. We do not know by which mechanism the chest could increase its sweat evaporation without increasing local sweat rate; however, the chest is a very vasculated region with high blood flow (Shvartz et al., 1979) and, therefore, if the acclimation protocol employed in the present study increases chest blood flow, more heat would be transferred from the blood to the skin and from the latter to the environment through sweat, leading to more sweat evaporation for a given sweat rate. Therefore, it is possible that in the present study some skin sites increased sweating efficiency (e.g., the chest) and others reduced it (e.g., the thigh) so that whole-body sweating efficiency was maintained equally throughout the acclimation period but with increased skin wettedness and sweat evaporation.

We found a higher core-to-skin gradient after the adaptation period partially related with the increased $\mathrm{E}_{\mathrm{sw}}(r=0.64, p=$ 0.009), representing a facilitated transport of heat from the core to the surface of the body and from the latter to the environment. This finding is corroborated by some (Candas et al., 1979b, Rowell et al., 1967) but not by others. (Patterson et al., 2004; Mitchell et al., 1976; Kenney et al., 1990). In the latter studies, a hot and humid environment was used, which limits sweat evaporation and, therefore, the reduction in skin temperature. Furthermore, wind speed, known to alter convective heat coefficient and, therefore, evaporative heat coefficient, was slower in those studies, which also limits sweat evaporation. We believe that those environmental conditions that differ from the present study could, at least in part, explain the difference in the core-to-skin gradient found.

Some studies have found a reduction in the concentration of sodium in the sweat following HA (Kirby and Convertino, 1986; Allan and Wilson, 1971; Nielsen et al., 1997), while others have not (Armstrong et al., 1989; Inoue et al., 1990). In the present study there was no change in local sweat sodium concentration after the acclimation period. Armstrong and Maresh (1991) suggested that sweat sodium concentration reduction occurs only when there is simultaneous diet sodium restriction, and that adaptation is an important factor for sodium conservation. The subjects of the present study maintained their regular diet and did not have any sodium restriction. In fact, they had a normal diet sodium content $(\sim 2000 \mathrm{mg} /$ day $)$ and this may be the reason for the nonsignificant change in sweat sodium concentration in the present study. Studying HA on Malaysian subjects, Saat et al. (2005b) observed higher sweat sodium concentration at rest after the protocol and a nonsignificant difference in sweat sodium concentration during exercise. It is known that sweat sodium concentration is influenced by sweat rate because electrolyte reabsorption is diminished due to augmented sweat rate (Saat et al., 2005b). To take this effect into account, we divided local sweat sodium concentration by local sweat rate. In fact, when relative to local sweat rate, sweat sodium concentration was reduced. This effect was recently observed by Buono et al. (2007). These authors observed that the $y$-intercept of the sweat sodium concentration with sweat rate was reduced, thus leading to a lower sweat sodium concentration for a given sweat rate following HA. Lower sweat sodium concentration is possibly brought by altered sweat gland responsiveness to aldosterone (Kirby and Convertino, 1986). The results found in the present study suggest that HA increased sodium reabsorption capacity of the sweat gland in tropical natives.

It could be speculated that the reduction in sweat sodium concentration found in the present study could partially account for the increase in sweat evaporation also observed. However, this seems unlikely since it was previously observed that the heat of evaporation of sweat with a much higher salt content does not differ from that of pure water (Snellen et al., 1970).

The fact that our subjects were tropical natives might have influenced some of the responses found in the present study. It has already been shown that tropical natives are more efficient thermoregulators as they rely less on sweat production and evaporation for heat dissipation than nontropical natives (Hori, 1995; Saat et al., 2005a). Evidence of the partial state of adaptation of the subjects of the present study is the nonsignificant change in resting rectal temperature after HA. In fact, there was a tendency to increase resting rectal temperature $(p=0.07)$. In agreement with this finding, Shapiro et al. (1981) found no difference in rectal temperature of individuals subjected to HA in the summer, while a significant reduction was found in the winter. Saat et al. (2005a) suggested that tropical subjects presented higher rectal temperature than temperate subjects during exercise, and Nguyen and Tokura (2003) speculated that this adaptation could be ecologically helpful in water saving.

In conclusion, our results show that HA improves sweat function in tropical natives. There was sweat redistribution towards the limbs without a concomitant change in the sweating efficiency. This redistribution correlated with an increase in the percentage of the skin covered by sweat, enhanced sweat evaporation, and higher thermoregulatory efficiency. 
Acknowledgments The authors are grateful to the following colleagues from the Exercise Physiology Laboratory: Maria Aparecida Vasconcelos Faria, for helping in data collection; and to Ana de Oliveira Rodrigues for extremely helpful advice on the final version of the manuscript. We also thank the volunteers for participating in the study. Capes, FAPEMIG, and CNPq, supported this study.

\section{References}

Allan JR, Wilson CG (1971) Influence of acclimatization on sweat sodium concentration. J Appl Physiol 30: 708-712

Armstrong LE, Francesconi RP, Kraemer WJ, Leva N, Deluca JP, Hubbard RW (1989) Plasma cortisol, rennin, and aldosterone during an intense heat acclimation program. Int J Sports Med 10: 38-42

Armstrong LE, Maresh CM (1991) The induction and decay of heat acclimatization in trained athletes. Sports Med 12: 302-312

Armstrong LE (2000) Performing in extreme environments. Human Kinetics, Champaign Belding HS, Hatch TF (1955) Index for evaluating heat stress in terms of resulting physiological strains. Heat Piping Air Cond 8:129-136

Berglund LG, Gonzalez RR (1977) Evaporation of sweat from sedentary man in humid environments. J Appl Physiol 42:767-772

Buono MJ, Ball KD, Kolkhorst FW (2007) Sodium ion concentration vs. sweat rate relationship in humans. J Appl Physiol 103: 990-994

Candas V, Libert JP, Vogt JJ (1979a) Human skin wettedness and evaporative efficiency of sweating. J Appl Physiol 46: 522-528

Candas V, Libert JP, Vogt JJ (1979b) Influence of air velocity and heat acclimation on human skin wettedness and sweating efficiency. J Appl Physiol 47: 1194-1200

Cotter JD, Patterson MJ, Taylor NAS (1997) Sweat distribution before and after repeated heat exposure. Eur J Appl Physiol 76: $181-186$

Dubois D, Dubois EF (1916) A formula to estimate the approximate surface area if height and weight be known. Arch Intl Med 17: 836-837

Ducharme MB, VanHelder WP, Radomski MW (1991) Tissue temperature profile in the human forearm during thermal stress at thermal stability. J Appl Physiol 71: 1973-1978

Fielding RA, Frontera WR, Hughes VA, Fisher EC, Evans WJ (1997) The reproducibility of the Bruce protocol exercise test for the determination of aerobic capacity in older women. Med Sci Sports Exerc 29: 1109-1113

Fox RH, Budd GM, Woodward PM, Hackett AJ, Hendrie AL (1974) A study of temperature regulation in New Guinea people. Phil Trans R Soc Lond B 268: 375-391

Fox RH, Goldsmith R, Hampton I FG, Lewis HE (1964) The nature of the increase in sweating capacity produced by heat acclimatization. J Physiol 171: 368-376

Fox RH, Goldsmith R, Hampton IFG, Hunt TJ (1967) Heat acclimatization by controlled hyperthermia in hot-dry and hot-wet climates. J Appl Physiol 22: 39-46

Fox RH, Goldsmith R, Kidd DJ, Lewis HE (1961) Acclimatization of the sweating mechanism in man. Proc Physiol Soc 14: 56P-57P

Frye AJ, Kamon E (1983) Sweating efficiency in acclimated men and women exercising in humid and dry heat. J Appl Physiol 54: 972-977

Hellon RF, Jones RM, Macpherson RK, Weiner JS (1956) Natural and artificial acclimatization to hot environments. J Physiol 132: 559 - 576

Höfler W (1968) Changes in regional distribution of sweating during acclimatization to heat. J Appl Physiol 25: 503-506

Hori S, Ihzuka H, Nakamura M (1976) Studies on physiological responses of residents in Okinawa to a hot environment. Jpn J Physiol 26: 235-244

Hori S (1995) Adaptation to heat. Jpn J Physiol 45: 921-946

Inoue Y, Havenith G, Kenney WL, Loomis JL (1999) Exerciseand methylcholine-induced sweating responses in older and younger men: effect of heat acclimation and aerobic fitness. Int J Biometeorol 42: 210-216

Kenney WL, Tankersley CG, Newswanger DL (1990) Age and hypohydration independently influence the peripheral vascular response to heat stress. J Appl Physiol 68:19021908

Kirby CR, Convertino VA (1986) Plasma aldosterone and sweat sodium concentrations after exercise and heat acclimation. J Appl Physiol 61: 967-970

Kolka MA, Holden WL, Gonzalez RR (1984) Heat exchange following atropine injection before and after heat acclimation. J Appl Physiol 56: 896-899

Kuno Y (1956) Human Perspiration. Charles C. Thomas Publisher, Springfield

Machado-Moreira CA, Magalhães FC, Vimieiro-Gomes AC, Lima NRV, Rodrigues LOC (2005) Effects of heat acclimation on sweating during graded exercise until exhaustion. J Therm Biol 30: 437-442

Magalhães FC, Machado-Moreira CA, Vimieiro-Gomes AC, Silami-Garcia E, Lima NRV, Rodrigues LOC (2006) Possible biphasic sweating response during a short-term hat acclimation protocol for tropical natives. J Physiol Anthropol 25: 215-219

Mitchell D, Senay LC, Wyndham CH, VanRensburg, AJ, Rogers GG, Strydom NB (1976) Acclimation in a hot, humid environment: energy exchange, body temperature, and sweating. J Appl Physiol 40: 768-778

Nadel ER, Pandolf KB, Roberts MF, Stolwijk JAJ (1974) Mechanisms of thermal acclimation to exercise and heat. J Appl Physiol 37: 515-520

Nguyen H, Tokura H (2003) Sweating and tympanic temperature during warm water immersion compared between Vietnamese and Japanese living in Hanoi. J Hum Ergol (Tokyo) 32: 9-16

Nielsen B, Hales JRS, Strange S, Juel Christensen N, Warberg J, Saltin B (1993) Human circulatory and thermoregulatory 
adaptations with heat acclimation and exercise in a hot, dry environment. J Physiol 460: 467-485

Nielsen B (1996) Olympics in Atlanta: a fight against physics. Med Sci Sports Exerc 28: 665-668

Patterson MJ, Stocks JM, Taylor NAS (2004) Humid heat acclimation does not elicit a preferential sweat redistribution towards the limbs. Am J Physiol (Regulatory Integrative Comp Physiol 286): R512-R518

Peter J, Wyndham CH (1966) Activity of the human eccrine sweat gland during exercise in a hot humid environment before and after acclimatization. J Physiol 187: 583 - 594

Porter AMW (1993) Sweat and thermoregulation in hominids. Comments prompted by publications of P. E. Wheeler 1984-1993. J Hum Evol 25: 417-423

Regan JM, MacFarlane DJ, Taylor NAS (1996) An evaluation of the role of skin temperature during heat adaptation. Acta Physiol Scand 158: 365-375

Roberts MF, Wenger CB, Stolwijk JAJ, Nadel ER (1977) Skin blood flow and sweating changes following exercise training and heat acclimation. J Appl Physiol 43: 133-137

Rowell LB, Kraning II KK, Kennedy JW (1967) Central circulatory responses to work in dry heat before and after acclimatization. J Appl Physiol 22:509-518

Saat M, Lee JB, Matsumoto T, Kosaka M, Ohwatari N, Motomura K, Kaneda E, Taimura A, Yamauchi M (1999) Relationship between the duration of stay in Japan of Malaysian subjects and the suppression of sweat gland sensitivity by iontophoretically applied acetylcholine. Acta Med Nagasaki 44: 49-53

Saat M, Sirisinghe RG, Singh R, Tochihara Y (2005b) Effects of short-term exercise in the heat on thermoregulation, blood parameters, sweat secretion and sweat composition of tropic-dwelling subjects. J Physiol Anthropol Appl Hum Sci 24: $541-549$

Saat M, Tochihara Y, Hashiguchi N, Sirisinghe RG, Fujita M, Chou CM (2005a) Effects of exercise in the heat on thermoregulation of Japanese and Malaysian males. J Physiol Anthopol Appl Hum Sci 24: 267-275

Shapiro Y, Hubbard RW, Kimbrough CM, Pandolf KB (1981) Physiological and hematologic responses to summer and winter dry-heat acclimation. J Appl Physiol (Respirat Environ Exercise Physiol) 50: 792-798

Shvartz E, Bhattacharya A, Spenrinde SJ, Brock PJ, Aciaraffa D, VanBeaumont W (1979) Sweating responses during heat acclimation and moderate conditioning. J Appl Physiol 46: 675-680

Snellen W, Mitchell D, Wyndham CH (1970) Heat of evaporation of sweat. J Appl Physiol 29: 40

Stolwijk JA, Hardy JD (1966) Partitional calorimetric studies of responses of man to thermal transients. J Appl Physiol 21: 967-977

Thomson ML (1954) A comparison between the number and distribution of functioning eccrine sweat glands in Europeans and Africans. J Physiol 123: 225-233

Vimieiro-Gomes AC, Magalhães FC, Amorim FT, MachadoMoreira CA, Rosa MS, Lima NRV, Rodrigues LOC (2005) Comparison of sweat rate during graded exercise and the local rate induced by pilocarpine. Braz J Med Biol Res 38: 1133-1139

Wyndham CH, Bouwer WVDM, Devine MG, Paterson HE (1952) Physiological responses of African laborers at various saturated air temperatures, wind velocities and rates of energy expenditure. J Appl Physiol 5: 290-298

Received: June 25, 2009

Accepted: October 7, 2009

Correspondence to: Luiz O. C. Rodrigues, Av. Antônio Carlos, 6627-Campus Pampulha, Belo Horizonte, Minas Gerais, CEP: 31310-250, Brazil

Phone: + 55-31-3499-2328

Fax: +55-31-3491-9460

e-mail: lor@ufmg.br 